2 Major Depressive Disorder Working Group of the Psychiatric GWAS Consortium. Mol Psychiatry 2013; 18: 497-511.

3 Ripke S, Sanders AR, Kendler KS, Levinson DF, Sklar P, Holmans PA et al. Nat Genet 2011; 43: 969-976.

4 Sklar P, Ripke S, Scott L, Andreassen OA, Cichon S, Craddock N et al. Nat Genet 2011; 43: 977-983.

5 Devlin B, Roeder K. Biometrics 1999; 55: 997-1004.

6 Richardson K, Lai CQ, Parnell LD, Lee YC, Ordovas JM. BMC Genomics 2011; 12: 504.

7 Gibbs JR, van der Brug MP, Hernandez DG, Traynor BJ, Nalls MA, Lai SL et al. PLoS Genet 2010; 6: e1000952.

81000 Genomes Project Consortium. Nature 2010; 467: 1061-1073.

Supplementary Information accompanies the paper on the Molecular Psychiatry website (http://www.nature.com/mp)

\section{Genes for endosomal NHE6 and NHE9 are misregulated in autism brains}

Molecular Psychiatry (2014) 19, 277-279; doi:10.1038/mp.2013.28; published online 19 March 2013

Autism is a highly heterogeneous neurodevelopmental disorder with impaired language, social communication, and restricted and repetitive interests and behavior. Monogenic developmental brain disorders with autism features such as Rett syndrome, Angelman syndrome, Fragile $\mathrm{X}$ syndrome, and others provide important tractable models of relevance to severe autism. In addition to observing autism symptoms in the monogenic condition, if the gene responsible is also significantly misregulated in the brains of people with idiopathic autism, then these data provide substantial independent support for the importance of the gene in autism pathophysiology.

Given this logic, we set out to test if there were gene expression changes in postmortem brain tissue from patients with idiopathic autism in the genes of interest that encode the $\mathrm{Na}^{+} / \mathrm{H}^{+}$ exchanger family of proteins, with particular interest in the forms of exchangers localized to endosomes, namely, NHE6 and NHE9. Mutations in the X-linked endosomal $\mathrm{Na}^{+} / \mathrm{H}^{+}$Exchanger 6 (NHE6, also known as SLC9A6) represent a novel neurogenetic syndrome with variable expressivity. ${ }^{1}$ In a systematic, large-scale resequencing screen of $X$-chromosome coding exons in $>200$ pedigrees consistent with $\mathrm{X}$-linked intellectual disability, NHE6 was among the top six most recurrently mutated genes. ${ }^{2}$ The 'Christianson syndrome', based on the initial clinical description, reported an association with autistic symptoms as has been reported subsequently. ${ }^{3,4}$ In parallel to the description of autistic symptoms associated with mutations in NHE6, Morrow et al. ${ }^{5}$ published mutations in the highly related endosomal protein NHE9 in severe autism with epilepsy. Endosomal processes, such as would be suggested by mutations in NHE6 and NHE9, represent an important cellular mechanism for investigations regarding disorders of cognitive development. Interestingly, of the six top genes implicated in the Tarpey et al. ${ }^{2}$ study, two of the genes, NHE6 and AP1S2, are known to be involved in endosomal mechanisms.

We therefore investigated whether the expression of $N H E$ genes, NHE6 and NHE9, were altered in autism cerebral cortex. We did this by renormalizing and analyzing publically available microarray data. Specifically, we first analyzed data from Voineagu et al., ${ }^{6}$ which were previously used to compare autism and control cortex ( $n=29$ of each). We renormalized the data as described in Voineagu et al., ${ }^{6}$ except for when we wished to analyze a subset of NHE genes that were excluded from the analysis because of low expression. Among their results, Voineagu et al. ${ }^{6}$ reported that synapse-associated genes were downregulated in autism cortex, ${ }^{6}$ and we confirmed this result by performing differential gene expression analysis ${ }^{7}$ between autism and control cortex (for detailed Methods, please see Supplementary Information). We then performed DAVID functional annotation clustering analysis ${ }^{8,9}$ on the 197 genes with at least 1.3-fold reduction in autism cortex and $P<0.05$ (after Benjamini-Hochberg adjustment ${ }^{10}$ ), which were the differential expression cutoffs described by Voineagu et al. ${ }^{6}$ Indeed, the most significant gene set in the top scoring cluster of the DAVID results was the Gene Ontology term 'synapse,' for which 21 genes overlapped with those downregulated in autism cortex (BH-adjusted overlap $P$-value $=2.2 \times 10^{-7}$ ). This confirmed that synapse-related genes, such as GABRA1 and CHRM1, were downregulated in autism cortex in the Voineagu et al. ${ }^{6}$ data set (Supplementary Tables 1 and 2 for description of the downregulated synapse genes). We next tested whether NHE genes (NHE1-11) were differentially expressed between autism and control cortex using a $t$-test (Supplementary Table 3). Notably, NHE1 and NHE6 were significantly downregulated in autism cortex $(P=0.0030$ and 0.0042 , respectively) and NHE9 was significantly upregulated $(P=0.00075)$, yet other NHE genes were not significantly differentially expressed between autism and control cortex $(P>0.15)$.

We further hypothesized that changes in these genes were reflective of broader changes in gene expression, such as downregulation of synapse genes. To investigate the functional changes associated with NHE1, NHE6 and NHE9 gene expression, we found Pearson correlation coefficients between each of these genes and the average expression of the 21 synapse-related genes. NHE1 was not significantly correlated with the synapse genes $(r=0.15, P=0.28)$, but NHE6 and NHE9 both were strongly correlated with the synapse genes $\left(r=0.86, P=4.2 \times 10^{-18}\right.$ for NHE6; $r=-0.62, P=2.2 \times 10^{-7}$ for NHE9—see Figure 1). Furthermore, the sub-population of samples with the lowest NHE6 expression was almost entirely autism cases, as was the subpopulation of high NHE9 expression. Additionally, NHE6 and NHE9 were negatively correlated $(P=0.00015)$, and when NHE6 expression was high, NHE9 was tightly regulated. A similar decrease in NHE9 variability could be seen with high synapse gene expression (Figure 1). Thus, a sub-population within autism is not only associated with lower synapse gene expression, but also with lower NHE6 expression and increased and potentially misregulated NHE9 expression. We also studied the correlation of NHE6 and NHE9 with the downregulated synapse gene set during normal brain development. We capitalized on the large mRNA-seq data set from human brain made available by the Allen Brain Institute (http://www.developinghumanbrain.org). We find that NHE6 clusters strongly during development with this synapse gene group (see Supplementary Information). NHE9 expression was far lower embryonically, increased postnatally but did not appreciably cluster with this synapse group in typical brain development (Supplementary Figures 1-3).

We used two independent microarray data sets for autism and control cerebral cortex to validate both the differential gene expression of NHE6 and NHE9 in autism cases and these genes' associations with synapse genes. The validation data sets were 

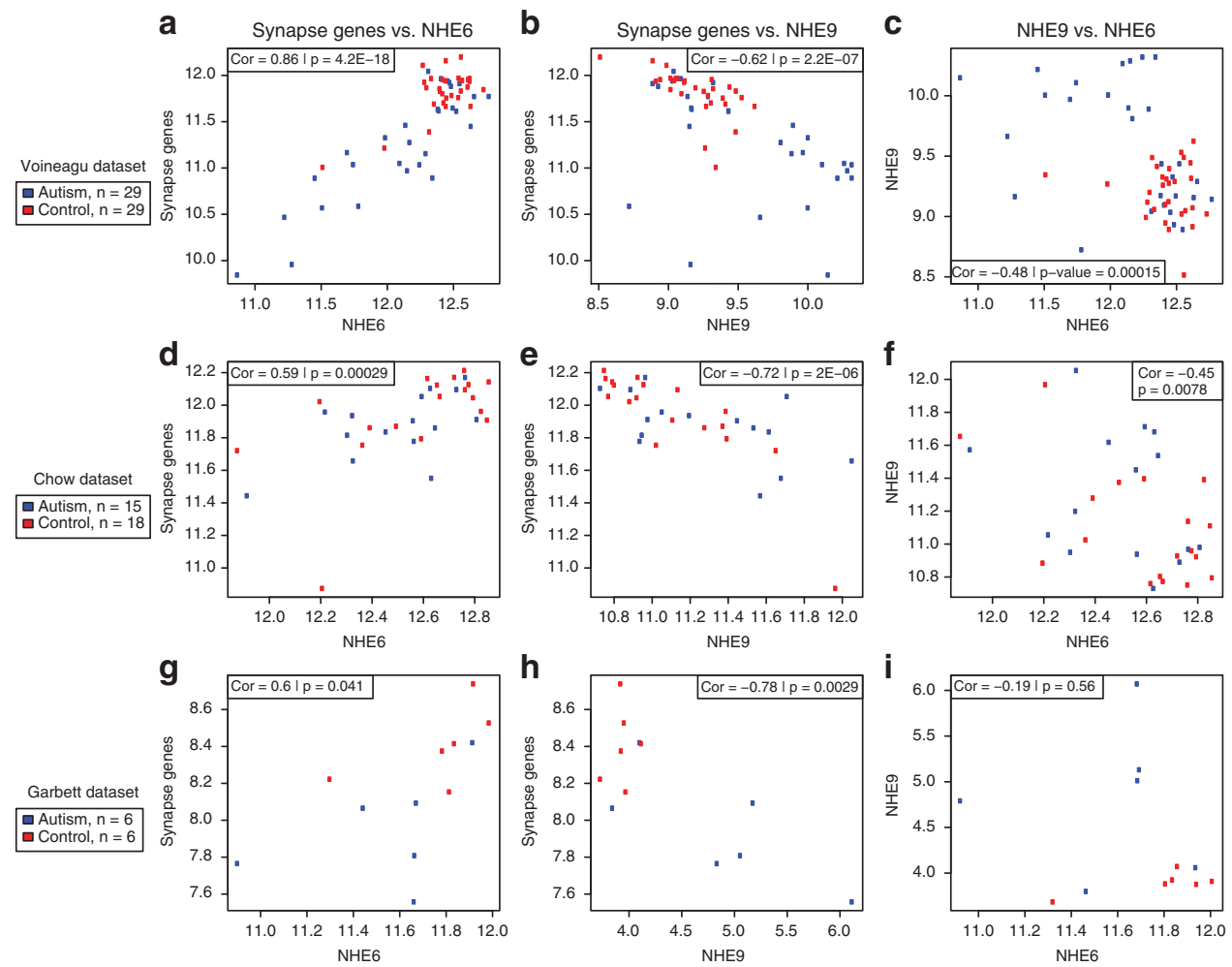

Figure 1. Expression plots of NHE6 versus synapse genes, NHE9 versus synapse genes and NHE6 versus NHE9 in three independent data sets. We compared the average log base 2 expression of 21 synapse genes with that of NHE6 and NHE9 expression in microarray data sets published by Voineagu et al. ${ }^{6}(\mathbf{a}-\mathbf{c}, n=58)$, Chow et al. ${ }^{11}(\mathbf{d}-\mathbf{f}, n=33)$ and Garbett et al. ${ }^{12}(\mathbf{g}-\mathbf{i}, n=12)$. Each data set was designed to compare gene expression in autism and control cerebral cortex. The synapse genes used were the 21 synapse genes downregulated in autism cortex compared with control in the Voineagu et al. ${ }^{6}$ data set (Supplementary Tables 1 and 2).

from Chow et al. ${ }^{11}(n=15$ autism, $n=18$ control) and Garbett et $a .^{12}$ ( $n=6$ of each; data set details in Supplementary Table 4). The Chow et al. ${ }^{11}$ data were downloaded from GEO (GSE28475), and the Garbett et al. ${ }^{12}$ data were provided by the authors. In the Garbett et al. ${ }^{12}$ data, NHE9 was significantly higher in autism than control cortex $(P=0.039)$, and while NHE6 was lower on average in autism cortex, this was not statistically significant $(P=0.22)$, although this may have been due to low sample size (Figure 1). In the Chow et al. ${ }^{11}$ data, although NHE6 and NHE9 had lower and higher expression in autism cortex compared with control, respectively, these trends were not significant $(P=0.38$ and 0.17). However, the mean expression of synapse genes was also not significantly different in the Chow et al. ${ }^{11}$ data set $(P=0.46)$, suggesting that the Voineagu et al. ${ }^{6}$ and Chow et al. ${ }^{11}$ data sets represent different populations. Additionally, NHE6 and NHE9 were negatively correlated across data sets, except for in Garbett et al., ${ }^{12}$ in which the correlation was not significant $(P=0.56)$ but suggesting as yet unknown mechanisms of interaction. Despite this lack of significance in the independent data sets, these genes' associations with synapse genes remained strong in both additional data sets: NHE6 is positively associated with synapse genes $\left(r=0.59, P=0.00029\right.$ for NHE6 in Chow et al. ${ }^{11}$ study; and $r=0.6, P=0.041$ for NHE6 in Garbett et al. ${ }^{12}$ study-see Figure 1) and NHE9 negatively associated $\left(r=-0.72, P=2.0 \times 10^{-6}\right.$ for NHE9 in Chow et al. ${ }^{11}$ study; and $r=-0.78, P=0.0029$ for NHE9 in Garbett et al. $^{12}$ study-see Figure 1). Finally, we tested for an association of gene expression for NHE6 and NHE9 and wellestablished autism-related genes such as SHANK2/3, NLGN4X, NRXN1 and PTEN. Notably, NHE6 and NHE9 each showed a strong association with NRXN1 $\left(P=2 \times 10^{-10}\right.$ for NHE6, and $P=2.9 \times 10^{-8}$ for NHE9; Supplementary Figures 4 and 5).
In summary, we find interesting gene expression changes in endosomal NHE6 and NHE9 in postmortem autism brains. These gene expression changes are largely replicated across data sets or the trends of these changes are maintained given limitations in sample size. We also report a strong correlation of endosomal NHE6 and NHE9 gene expression with the synapse genes across all data sets. The strong correlation of endosomal NHEs with synapse genes suggests that changes in synapse genes in autism involves a cellular mechanism that involves endosomal NHE6 and NHE9 in at least some autism brains. In conclusion, these gene expression studies in postmortem brains from patients with idiopathic autism provide additional support, in addition to the association of autism symptoms with the single-gene mutations, that endosomal NHEs are mechanistically involved in the pathophysiology of autism.

\section{CONFLICT OF INTEREST}

The authors declare no conflict of interest.

\section{ACKNOWLEDGEMENTS}

EMM has received a Career Award in Medical Science from the Burroughs Wellcome Fund and support from NIMH 1K23MH080954-05. This work was supported by a grant from the Simons Foundation (SFARI \#239834 to EMM), and also generous support to EMM from the Nancy Lurie Marks Foundation.

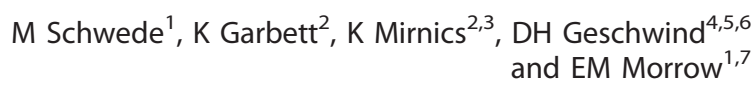

${ }^{1}$ Department of Molecular Biology, Cell Biology and Biochemistry, Institute for Brain Science, Brown University, Laboratory for Molecular Medicine, Providence, RI, USA 
${ }^{2}$ Department of Psychiatry, Vanderbilt University, Nashville, TN, USA;

${ }^{3}$ Vanderbilt Kennedy Center for Research on Human Development, Vanderbilt University, Nashville, TN, USA;

${ }^{4}$ UCLA Center for Autism Research and Treatment, Semel Institute for Neuroscience and Behavior, Los Angeles, CA, USA;

${ }^{5}$ Program in Neurogenetics, Department of Neurology, David Geffen School of Medicine at UCLA, Los Angeles, CA, USA; ${ }^{6}$ Department of Human Genetics, David Geffen School of Medicine at UCLA, Los Angeles, CA, USA and

${ }^{7}$ Department of Psychiatry and Human Behavior, Emma Pendleton Bradley Hospital, Alpert Medical School of Brown University,

East Providence, RI, USA

E-mail: eric_morrow@brown.edu

\section{REFERENCES}

1 Gilfillan GD, Selmer KK, Roxrud I, Smith R, Kyllerman M, Eiklid K et al. Am J Hum Genet. 2008; 82: 1003-1010.

2 Tarpey PS, Smith R, Pleasance E, Whibley A, Edkins S, Hardy C et al. Nat Genet. 2009; 41: 535-543.

3 Garbern JY, Neumann M, Trojanowski JQ, Lee VM, Feldman G, Norris JW et al. Brain 2010; 133(Pt 5): 1391-1402.

4 Christianson $\mathrm{AL}$, Stevenson RE, van der Meyden $\mathrm{CH}$, Pelser J, Theron FW, van Rensburg PL. J Med Genet. 1999; 36: 759-766.

5 Morrow EM, Yoo SY, Flavell SW, Kim TK, Lin Y, Hill RS et al. Science 2008; 321: 218-223.

6 Voineagu I, Wang X, Johnston P, Lowe JK, Tian Y, Horvath S et al. Nature. 2011; 474: $380-384$.

7 Smyth GK. Stat Appl Genet Mol Biol. 2004; 3: Article 3.

8 Huang da W, Sherman BT, Lempicki RA. Nucleic Acids Res. 2009; 37: $1-13$.

9 Huang da W, Sherman BT, Lempicki RA. Nat Protoc. 2009; 4: 44-57.

10 Benjamini Y, Drai D, Elmer G, Kafkafi N, Golani I. Behav Brain Res. 2001; 125: 279-284.

11 Chow ML, Pramparo T, Winn ME, Barnes CC, Li HR, Weiss L et al. PLoS Genet. 2012; 8: e1002592.

12 Garbett K, Ebert PJ, Mitchell A, Lintas C, Manzi B, Mirnics K et al. Neurobiol Dis. 2008; 30: 303-311.

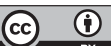

This work is licensed under a Creative Commons Attribution 3.0 Unported License. To view a copy of this license, visit http:// creativecommons.org/licenses/by/3.0/

Supplementary Information accompanies the paper on the Molecular Psychiatry website (http://www.nature.com/mp)

\section{Amphetamine-induced} behavior requires CaMKII-dependent dopamine transporter phosphorylation

Molecular Psychiatry (2014) 19, 279-281; doi:10.1038/mp.2013.29; published online 19 March 2013

The dopamine (DA) transporter (DAT), which mediates the inactivation of released DA through its reuptake, is a primary molecular target for psychostimulants. ${ }^{1,2}$ Cocaine and methylphenidate (MPH) exert their psychostimulant properties by blocking DA reuptake, leading to the elevation of extracellular DA. ${ }^{2}$ In contrast, amphetamine (AMPH) acts as a substrate for DAT and subsequently induces non-exocytotic DAT-mediated release of DA (DA efflux). ${ }^{2}$ Here we use a Drosophila behavioral assay to delineate the signaling mechanisms that modulate DAT-mediated $\mathrm{AMPH}$-induced behavior in vivo. Understanding these mechanisms is critical to understanding how the actions of AMPH might be blocked therapeutically, while simultaneously preserving DA transport.

Multiple lines of evidence suggest that kinase activity can modulate DAT function and, more specifically, AMPH action on DAT. Both calcium/calmodulin kinase II alpha (CaMKII) and protein kinase C (PKC) can phosphorylate an N-terminal DAT peptide in vitro $^{3}$ and inhibiting the activity of either kinase attenuates AMPH-induced DA efflux in rodent striatial slices. ${ }^{3,4}$ Data from CaMKII and PKC knockout mice suggest a role for these kinases in regulating $\mathrm{AMPH}$ action in vivo, ${ }^{5,6}$ However, it is difficult to ascertain from these studies using global knockout strategies whether the observed effects on AMPH-induced DA efflux are mediated directly through alterations in DAT phosphorylation or whether they arise indirectly through phosphorylation of other targets or through circuit effects.

Recently we showed that phosphorylation of the N-terminus of DAT is essential for AMPH-induced, but not MPH-induced hyperlocomotion in Drosophila. Larvae respond to either AMPH or MPH by increasing their crawling velocity, and a null mutation in Drosophila DAT $\left(d D A T^{f m n}\right)$ abolishes these locomotor responses. ${ }^{7}$ Expression of wild-type human (hDAT) in DA neurons of $d D A T^{f m n}$ mutants rescues the response to either psychostimulant. ${ }^{7}$ In contrast, expression of a phospho-deficient mutant hDAT (hDAT-S/A) rescued only the response to MPH but not to $\mathrm{AMPH}^{7}$ consistent with studies showing that DAT phosphorylation is required for $\mathrm{AMPH}$-induced $\mathrm{DA}$ efflux but not for DA reuptake in heterologous cultured cells. ${ }^{3,8}$ Using the tractable Drosophila system, we have now determined whether CaMKII is required specifically in DA neurons for AMPH-induced behavior. To inhibit the activity of CaMKII, we used a UAS-driven highly selective inhibitory peptide (CaMKIINtide). ${ }^{9}$ We expressed CaMKIINtide in DA neurons of larvae using the tyrosine hydroxylase (TH) GAL4 driver. ${ }^{10}$ These larvae were fed either vehicle or AMPH and their speed of locomotion was measured as previously described. ${ }^{7}$ Larvae expressing CaMKIINtide in DA neurons (UASCaMKIINtide(2x)/TH-GAL4) failed to increase their crawling velocity in response to AMPH in contrast to control larvae without TH-Gal4 (UAS-CaMKIINtide $(2 x) /+$ ), which exhibited significant hyperlocomotion (Figure 1a). Expression of CaMKIINtide in DA neurons did not inhibit MPH-induced hyperlocomotion (Figure 1b), consistent with our previous finding that phosphorylation of DAT is not required for the behavioral response to this DA uptake inhibitor. ${ }^{7}$ We also found that co-expression of CaMKIINtide with hDAT in DA neurons of mutant larvae that lack dDAT (dDAT ${ }^{f m n}$; TH-GAL4,UAShDAT/UAS-CaMKIINtide(2x)) blunted AMPH-induced but not MPHinduced hyperlocomotion (Figures $1 \mathrm{c}$ and $\mathrm{d}$, respectively). These data show that CaMKII is essential specifically in DA neurons for both dDAT-mediated and hDAT-mediated AMPH-induced hyperlocomotion in Drosophila. They also suggest that CaMKII activity is not required for DA uptake in vivo, as the response to MPH was unaffected in its absence.

To examine whether the role of CaMKII is mediated via DAT phosphorylation, we determined whether pseudophosphorylation of the DAT N-terminus can decouple the AMPH-induced behavior from the activation state of CaMKII. Previously we demonstrated that a mutant hDAT (hDAT-S/D), which mimics constitutive phosphorylation by mutation of the five $\mathrm{N}$-terminal serines to aspartates, could restore AMPH-induced hyperlocomotion in $\mathrm{dDAT}$ mutant larvae. ${ }^{7}$ When we expressed hDAT-S/D in animals where CaMKII was inhibited (dDAT $T^{f m n}$; TH-GAL4, UAS-hDAT-S/D/UASCaMKIINtide $(2 x)$ ) we found that the response to AMPH was restored (Figure 1e). In contrast, larvae expressing wild-type hDAT (dDAT ${ }^{f m n}$; TH-GAL4,UAS-hDAT/UAS-CaMKIINtide(2x)) failed to respond to AMPH (Figure 1e). These data, combined with in vitro studies that show that CaMKII associates with the C-terminus of DAT and phosphorylates an N-terminal DAT peptide, ${ }^{3}$ suggest that 